\title{
Elevated Anaphylatoxin C3a in Expressed Prostatic Secretion Correlates with Prostatic Inflammation Extent in Benign Prostatic Hyperplasia
}

\author{
HaichaoYuan, Benlin Wei, Liangchao Ni, Yongqing Lai, Shangqi Yang* \\ Department of Urology, Peking University Shenzhen Hospital, Lianhua Road \#1120, Shenzhen, Guangdong, \\ P.R. China \\ *Corresponding Author:Shangqi Yang, Department of Urology, Peking University Shenzhen Hospital, \\ Lianhua Road\#1120, Shenzhen, Guangdong, P.R. China, Email:916459069@qq.com
}

\begin{abstract}
Objective: To investigate the possible association between the anaphylatoxin C3a levels in expressed prostatic secretion (EPS) and the extent of prostatic inflammation to preliminarily speculate whether the anaphylatoxin C3a contributes to the progression of benign prostatic hyperplasia (BPH).
\end{abstract}

Methods: Forty patients with BPH undergoing transurethral resection of the prostate (TURP) were studied. The EPS was collected for the measurement of anaphylatoxin C3a levels by enzyme-linked immunosorbent assays (ELISA). According to the grades of prostatic inflammatory infiltrates, the patients were divided into mild and moderate-severe groups to compare the C3a concentrations in the EPS. The correlations between anaphylatoxin C3a levels and BPH related clinical factors, such as age, prostate volume and urine WBC, were analyzed. A receiver-operating characteristic (ROC) curve was used to analyze the anaphylatoxin C 3 a levels in the EPS as a screening indicator in the diagnosis of the prostatic inflammation extent in BPH patients.

Results: A total of 232/240 (96.6\%) BPH patients had chronic prostatitis with predominant periglandular inflammation. Significantly increased levels of $\mathrm{C} 3$ a were observed in the moderate-severe group compared with the mild group (mean C3a $638.78 \mathrm{ng} / \mathrm{mL}$ vs. $440.74 \mathrm{ng} / \mathrm{mL}$ ). No statistically significant associations were found between the anaphylatoxin C3a levels and the age, prostate volume or urine WBC. BPH with moderatesevere chronic prostatitis and mild chronic prostatitis achieved at the established C3a cut-point was 392 $n g / m L$.

Conclusions: Anaphylatoxin C3a levels in the EPS are a relative reliable biomarker for evaluating the prostatic inflammation extent in BPH patients and might be helpful for monitoring the effects of antiinflammatory therapies in further studies.

\section{INTRODUCTION}

Benign prostatic hyperplasia $(\mathrm{BPH})$ represents the most common urologic disease among elderly males and has been a significant health concern in the aging society ${ }^{1}$. The pathogenesis of BPH remains largely unresolved. Several recent studies have shown that $\mathrm{BPH}$ is frequently associated with chronic inflammatory infiltrates that are mainly composed of $\mathrm{T}$ and $\mathrm{B}$ lymphoid cells and macrophages. Proinflammatory cytokine interleukins (IL-1, IL-2, IL-6, IL-8, IL-15 and IL-17) can be found. Prostatic epithelial cells and stromal cells express members of the toll-like receptor family and are capable of recognizing foreign incoming antigens $^{2}$.

Like the intestine or the lung, the prostate could be considered an immune competent organ. Deregulation of the immune response might occur in the prostate, inducing prostatic disease. BPH might be an immune inflammatory disease $^{3}$. The complement system is an important component of the immune inflammatory response $\mathrm{e}^{4-6}$. We hypothesized that this system might play an important role in the pathogenesis of BPH.

Anaphylatoxin C3a is a small peptide generated by complement activation. Anaphylatoxin C3a 
acts as a potent chemoattractant and a vasodilator, inducing smooth muscle contraction and histamine release $\mathrm{s}^{4,5}$. More recent studies have revealed that, in addition to its traditional roles in inflammation, anaphylatoxin C3a significantly influences the adaptive immune response $^{4-6}$. These studies suggested that C3a interacting with its receptor $\mathrm{C} 3 \mathrm{aR}$ in macrophages can up-regulate macrophage function to promote Th1 immune responses ${ }^{5}$. Anaphylatoxin plays an important role in regulating the innate and adaptive immune inflammatory responses.

In our study, we analyzed the anaphylatoxin C3a in the expressed prostatic secretion (EPS) in BPH patients, and we attempted to investigate the possible association between the $\mathrm{C} 3 \mathrm{a}$ levels in EPS and the extent of prostatic inflammation to preliminarily speculate whether anaphylatoxin $\mathrm{C} 3 \mathrm{a}$ contributes to the pathogenesis and diagnosis of BPH.

\section{MeThODS}

\subsection{Participants}

A total of 240 consecutive patients diagnosed with BPH were recruited to participate in the study betweenSeptember 2011 and March 2015. Participants were excluded if they had a preoperative diagnosis of prostatic carcinoma, previous prostatic surgery, symptoms related to prostatitis or a history of a documented urinary tract infection. All of the BPH patients were scheduled for transurethral resection of the prostate (TURP). This study was approved by the Medical Ethics Committee of Peking University Shenzhen Hospital. All of the participants provided written informed consent before participating in the study.

\subsection{Clinical and Laboratory Data Collection}

The clinical data, including the age, serum PSA, concomitant disease and medical history were obtained. A urinalysis was performed before the TURP and was regarded as abnormal when the white blood cell (WBC) count exceeded five per high power field (HPF). For the International Prostate Symptom Score (IPSS), a validated eight-item questionnaire was used to assess lower urinary tract symptoms (LUTS) (7). All of the participants underwent digital rectal examination and transrectal ultrasonography. The prostate volume (PV) was calculated using the following formula: $0.52 \times$ anterior-posterior diameter $(\mathrm{cm}) \times$ transverse diameter $(\mathrm{cm})$ $\times$ superior-inferior diameter $(\mathrm{cm}) \quad(8)$. The Medical Therapy of Prostatic Symptoms (MTOPS) Study (9) suggested that age $\geq 62$ years or $\mathrm{PV} \geq 31 \mathrm{~mL}$ were relative influencing factors for which BPH patients are most likely have clinical progression. Based on this, the patients were grouped. The EPS was obtained by prostatic massage (PM) and then collected to a cryovial by a sterile operation immediately before the TURP. The EPS was stored at $-20{ }^{\circ} \mathrm{C}$ for approximately $2 \mathrm{~h}$ and then transferred into a refrigerator for storage at $-80{ }^{\circ} \mathrm{C}$ until the EPS was used for cytokine analyses.

\subsection{The Diagnostic Standard of the Extent of Prostatic Inflammation in BPH}

During the TURP, the resected prostatic tissues, bigger and less burning were collected under sterile conditions, and they were washed repeatedly with sterile normal saline. The samples were fixed in 10\% neutral-buffered formalin and embedded in paraffin following routine histologic techniques. The sections (5 $\mu \mathrm{m})$ were stained with hematoxylin and eosin (HE). According to the North American Chronic Prostatitis Collaborative Research Network and International Prostatitis Collaborative Network (10), the grades of the prostatic inflammatory infiltrates are mild, moderate or severe, and the anatomical locations are periglandular, glandular and stromal. Our experienced pathologist adopted the histopathological classification system for chronic prostatic inflammation in BPH tissues.

\subsection{The Expression of C3a in EPS Examined by ELISA}

The anaphylatoxin C3a levels were measured using commercial enzymelinkedimmunosorbent assay (ELISA) kits (Amersham, Buckinghamshire, UK). These assays use the quantitative sandwich enzyme immunoassay technique and are specific for C3a, with a minimum detectable dose of 5 $\mathrm{pg} / \mathrm{mL}$. The assays were optically read at 450 $\mathrm{nm}$ in an ELISA plate reader (Bio-Rad Laboratories, Hercules, CA) within 30 min. The $\mathrm{C} 3 \mathrm{a}$ concentrations were determined by 
comparisons with a standard curve with a fiveparameter curve-fit analysis program.

\subsection{Statistical Analysis}

All of the analyses were performed on SPSS 13.0 for Windows (SPSS Inc., Chicago, IL, USA). The continuous variables were represented as the mean \pm standard deviation (SE), and the categorical data were represented by the number and percentage. Differences among two or more than two groups were assessed by a one-way analysis of variance test. Pearson correlation analysis was used to assess the correlations between the groups. A receiveroperating characteristic (ROC) curve was used to analyze the C3a levels in the EPS as a screening indicator in diagnosis of the extent of prostatic inflammation in $\mathrm{BPH}$ patients. $\mathrm{P}<0.05$ was defined as significant.

\section{Results}

Of the BPH patients included in the study, 232/240 (96.6\%)had chronic prostatitis (CP). The general characteristics of the enrolled patients are shown in Table 1. There were no statistically significant associations found between the extent of prostatic inflammation and the mean age, PSA, total IPSS or prostate volume $(\mathrm{P}=.076, \mathrm{P}=.531, \mathrm{P}=.306$ and $\mathrm{P}=.142$, respectively) (Table 1).To evaluate the effect of the related clinical factors, the age $(\geq 62 \mathrm{y} ;<62$ $\mathrm{y})$, prostate volume $(\geq 31 \mathrm{~mL} ;<31 \mathrm{~mL})$ and urine WBC $(\geq 5 / \mathrm{HP} ;<5 / \mathrm{HP})$ and the $\mathrm{C} 3 \mathrm{a}$ concentrations in the EPS were compared (Table 2). No statistically significant differences were found $(\mathrm{P}=.138, \mathrm{P}=.209, \mathrm{P}=.925$, respectively.

Table1.Baseline characteristics of the BPH with CP patients

\begin{tabular}{|l|l|l|l|}
\hline Characteristic & Mild & Moderate-Severe & P \\
\hline Patient (n) & 138 & 102 & .076 \\
\hline Mean Age (years) & $72.80 \pm 12.04$ & $70.10 \pm 7.12$ & .531 \\
\hline PSA (ng/mL) & $4.79 \pm 1.96$ & $5.51 \pm 4.20$ & .306 \\
\hline Total IPSS & $22.04 \pm 4.12$ & $20.50 \pm 5.15$ & .142 \\
\hline Prostate volume (mL) & $55.41 \pm 26.09$ & $46.78 \pm 22.23$ & .001 \\
\hline C3a (ng/ml) & $440.74 \pm 161.15$ & $638.78 \pm 166.56$ & \\
\hline
\end{tabular}

BPH=benign prostatic hyperplasia; $C P=$ chronic prostatitis; $P S A=$ prostate specific antigen; IPSS= International Prostate Symptom Score.

All values are mean \pm SE; $P<0.05$ was defined as significant.

Table2. The relationships between C3a level in EPS and related clinical factors

\begin{tabular}{|c|c|c|c|c|}
\hline clinical factors & Groups & Numbers & C3a $(\mathbf{n g} / \mathbf{m L})$ & P \\
\hline \multirow{2}{*}{ Age } & $\geq 62 \mathrm{y}$ & 174 & $548.55 \pm 101.56$ & .138 \\
\cline { 2 - 5 } & $<62 \mathrm{y}$ & 66 & $422.64 \pm 149.09$ & \\
\hline \multirow{2}{*}{ prostate volume } & $\geq 31 \mathrm{~mL}$ & 192 & $533.32 \pm 175.61$ & .209 \\
\cline { 2 - 5 } & $<31 \mathrm{~mL}$ & 48 & $436.36 \pm 251.50$ & .925 \\
\hline \multirow{2}{*}{ urine WBC } & $\geq 5 / \mathrm{HP}$ & 42 & $502.28 \pm 201.59$ & \\
\cline { 2 - 5 } & $<5 / \mathrm{HP}$ & 198 & $512.58 \pm 251.50$ & \\
\hline
\end{tabular}

EPS = expressed prostatic secretion; WBC=White blood cells; HP=high power field.

All values are mean $\pm S E ; P<0.05$ was defined as significant.

For the grades of the extent of prostatic inflammation, the $\mathrm{C} 3$ a concentration differences in the EPS were analyzed. Significantly increased levels of $\mathrm{C} 3 \mathrm{a}$ were observed in $\mathrm{BPH}$ patients with moderate-severe chronic prostatitis compared with BPH patients with mild chronic prostatitis (mean C3a 638.78 ng/mL vs. 440.74 $\mathrm{ng} / \mathrm{mL}, \mathrm{P}=.001)$. These results suggested that the C3a concentration in the EPS could be a biomarker for evaluating the extent of prostatic inflammation in BPH patients.

A receiver operating characteristic (ROC) curve was used to analyze the C3a level in the EPS as a screening indicator in the diagnosis of the extent of prostatic inflammation in $\mathrm{BPH}$ patients. The largest relative sensitivity and specificity for distinguishing $\mathrm{BPH}$ with moderate-severe chronic prostatitis and mild 
chronic prostatitis achieved at the established respectively (Fig. 1). C3a cut-point of $392 \mathrm{ng} / \mathrm{mL}$ were $94 \%$ and $45 \%$,

\section{R OC Curve}

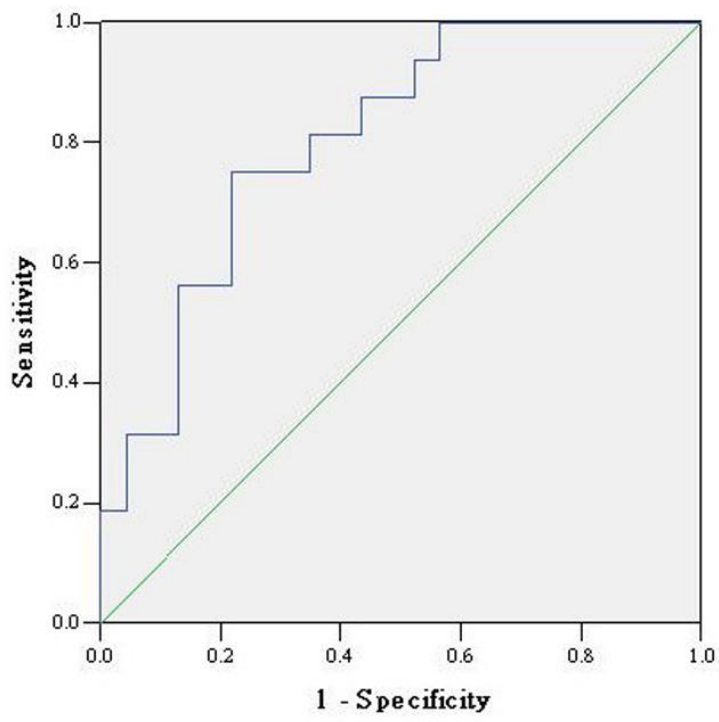

Figure1.The ROC curve of the anaphylatoxin C3a levels in EPS for the prostatic inflammation extent (mild and moderate-severe) in BPH patients with CP (AUC=0.804, 95\% CI (confidence interval) [0.669-0.940]).

\section{DISCUSSION}

The complement system consists of a tightly regulated network of proteins that play an important role in host defense and inflammation. Inappropriate complement activation and complement deficiencies are the underlying cause of the pathophysiology of many diseases, such as systemic lupus erythematous and asthma in $\mathrm{BPH}^{4,5}$, evidence indicates that chronic immune inflammation has a crucial role in the development of the disease, but correlational studies between the complement system and $\mathrm{BPH}$ are rare. Doble A et al. demonstrated that complement (C3) deposition in prostatic tissue was significantly correlated with poor urinary flow, irritative voiding and urgency ${ }^{11}$. Yi FX et al. ${ }^{12}$ found that a high concentration of $\mathrm{C} 3$ in the $\mathrm{PF}$ is a risk factor for the extent of prostatic inflammation and prostatic infections $(\mathrm{P}=0.035$ and 0.003 , respectively). Our study showed, for the first time, a correlation between the levels of $\mathrm{C} 3 \mathrm{a}$ in the EPS and the extent of prostatic inflammation in $\mathrm{BPH}$ patients.

Anaphylatoxin $\mathrm{C} 3 \mathrm{a}$ is a small peptide with a molecular mass of approximately $10 \mathrm{kDa}$. It consists of 76 amino acids ${ }^{13}$. Anaphylatoxin $\mathrm{C} 3 \mathrm{a}$ occurs in a variety of cells (e.g., monocytes/macrophages, neutrophils, mast cells, endothelial cells and epithelial cells) and tissues/organs (e.g., lung, kidney) constitutively or in response to various stimuli (infectious or non-infectious).

In our study, the association between the $\mathrm{C} 3 \mathrm{a}$ levels in the EPS and the extent of prostatic inflammation was significant. With increased levels of C3a in the EPS increased, there was an increased extent of prostatic inflammation in $\mathrm{BPH}$ with $\mathrm{CP}$. We found that related clinical factors, such as age, prostate volume and abnormal WBC count in the urinalysis, had no significant relationship with the $\mathrm{C} 3 \mathrm{a}$ levels in the EPS. These factors may have little impact on its levels.

By ROC curve analysis, we discovered that with C3a levels up to $392 \mathrm{ng} / \mathrm{mL}$, approximately $94 \%$ of BPH patients might have moderate-severe chronic prostatitis. The results described earlier indicated that the $\mathrm{C} 3 \mathrm{a}$ concentration in EPS could be a biomarker to preliminarily evaluate the extent of prostatic inflammation in $\mathrm{BPH}$ patients.

Because prostatic inflammation is associated with prostate enlargement and symptomatic $\mathrm{BPH}$, inflammation may be considered a therapeutic target in BPH (14). Some antiinflammatory agents have been tested in vitro and in vivo for the treatment of BPH. These anti-inflammatory agents include ibuprofen, cyclooxygenase-2 (COX-2) inhibitor 
(celecoxib), diclofenac, phytotherapy (Serenoarepens) ${ }^{15-19}$. These agents were found to achieve better therapeutic effects in BPH related symptoms. For BPH patients with inflammation, we should advocate for antiinflammatory therapies and close surveillance, but reliable biomarkers have not yet been validated to detect prostatic inflammation in routine clinical practice. Serum malondialdehyde (MDA), serum $\mathrm{C}$ reactive protein (CRP) and IL-8 in PF have been proposed as indicators in some studies ${ }^{20-22}$.

In present study, the $\mathrm{C} 3 \mathrm{a}$ levels were measured using ELISA. This technology has the advantages of lower cost and easy operation. Measuring the C3a levels in the local EPS instead of in serum could reduce the interference of various factors and more accurately reflect the local prostatic microenvironment. The $\mathrm{C} 3 \mathrm{a}$ concentration in EPS may be helpful for the preliminary evaluation of the extent of prostatic inflammation in BPH patients and for monitoring the effects of anti-inflammatory therapies and adjusting the therapeutic regimen in routine clinical practice.

Anaphylatoxin C3a had been traditionally known to induce smooth muscle contraction and the histamine release from mast cells and for enhanced vascular permeability. It mediates chemotaxis, inflammation and the generation of cytotoxic oxygen radicals. Anaphylatoxin C3a plays important roles in the induction and regulation of the innate immune inflammation responses. Recent studies suggest that anaphylatoxin $\mathrm{C} 3 \mathrm{a}$ acts through its on its receptor $\mathrm{C} 3 \mathrm{aR}$, which belongs to the G-protein coupled receptor family and is expressed on innate immune cells such as macrophages, to regulate the adaptive immune response and significantly influence immunological diseases ${ }^{4-}$ ${ }^{6}$. Zhou et al. ${ }^{23}$ showed that peritoneal macrophages derived from $\mathrm{C} 3$ deficient mice (which are unable to generate $\mathrm{C} 3 \mathrm{a}$ ) had a lowered surface expression of MHC class II and a reduced ability to elicit all specific Th1 responses in vitro and in vivo. That suggested that $\mathrm{C} 3 \mathrm{a}$ interacting with its receptor in macrophages can up-regulate macrophage function to promote Th1 immune responses. Macrophages can make up $15 \%$ of the inflammatory infiltrate in $\mathrm{BPH}$. We suspect that increased anaphylatoxin C3a levels in the EPS might lead to increased macrophage infiltration in $\mathrm{BPH}$ tissue. Macrophage infiltration might promote Th1 immune responses by up- regulation of the MHC class II antigen human leukocyte antigen (HLA)-DR, which has been demonstrated in $\mathrm{BPH}{ }^{24}$. The persistence of inflammation in the prostatic tissue might cause the anaphylatoxin C3a to continuously stimulate macrophages, those changed prostatic microenvironment and might cause T-cell aggregation and inflammatory cytokine release. The stromal-epithelial interactions eventually lead to the development and growth of the prostate gland and $\mathrm{BPH}$.

We speculated that anaphylatoxin C3a might play an important role in the occurrence and development of prostatic inflammation and might contribute to the pathogenesis of $\mathrm{BPH}$. We do not currently know much about the precise role of anaphylatoxin $\mathrm{C} 3 \mathrm{a}$ in prostatic tissue. Novel treatment strategies, such as individual immune regulation, should be considered.

\section{Conclusions}

Anaphylatoxin $\mathrm{C} 3 \mathrm{a}$ as an important component of the complement system, and its levels in EPS might be a reliable biomarker for evaluating the extent of prostatic inflammation in BPH patients and for preliminarily monitoring the effects of anti-inflammatory therapies. By regulating the innate and adaptive immune response, anaphylatoxin $\mathrm{C} 3$ a might play an important role in the pathogenesis and progression of BPH and chronic prostatitis. Our results require future prospective studies with larger samples. These studies are needed to confirm our results and to explore the precise mechanism of anaphylatoxin $\mathrm{C} 3 \mathrm{a}$ in the pathogenesis and progression of prostatic diseases.

\section{ACKNOWLEDGEMENTS}

The present study was supported by grants from Scientific research projects of Health and family planning system of Shenzhen (grant no.SZBC2017021). SQY conceived and designed the experiments. HCY and BLW performed the experiments. HCY produced the manuscript. HCY and LCN conducted data analysis.

\section{REFERENCES}

[1] Alcaraz A, Hammerer P, Tubaro A, et al. Is there evidence of a relationship between benign prostatic hyperplasia and prostate cancer? Findings of a literature review. EurUrol 2009; 55:864-875.

[2] Bob Djavan, Elisabeth Eckersberger, Geovanni Espinosa, et al. Complex Mechanisms in 
Prostatic Inflammatory Response. EurUrolSuppl 2009; 8:872-878.

[3] Kramer G, Mitteregger D, Marberger M. Is benign prostatic hyperplasia (BPH) an immune inflammatory disease? EurUrol 2007;51: 12021216.

[4] Sarma JV, Ward PA. Ward. The complement system. Cell Tissue Res 2011; 343:227-235.

[5] Zhou, W. The new face of anaphylatoxins in immune regulation. Immunobiology 2012; 217:225-234.

[6] Peng Q, Li K, Sacks SH, et al. The role of anaphylatoxins $\mathrm{C} 3 \mathrm{a}$ and $\mathrm{C} 5 \mathrm{a}$ in regulating innate and adaptive immune responses. Inflamm Allergy Drug Targets 2009; 8:236246.

[7] Penna G, Mondaini N, Amuchastegui S, et al. Seminal plasma cytokines and chemokines in prostate inflammation: interleukin 8 as a predictive biomarker in chronic prostatitis/chronic pelvic pain syndrome and benign prostatic hyperplasia. EurUrol 2007; 51:524-533.

[8] Tang P, Du W, Xie K, et al. Transition zone PSA density improves the prostate cancer detection rate both in PSA 4.0-10.0 and 10.1$20.0 \mathrm{ng} / \mathrm{ml}$ in Chinese men. Urologic Oncology: Seminars and Original Investigations 2011, doi:10.1016/j.urolonc.2011.06.012.

[9] McConnell JD, Roehrborn CG, Bautista OM, et al. The long-term effect of doxazosin, finasteride, and combination therapy on the clinical progression of benign prostatic hyperplasia. N Engl J Med. 2003; 349:23872398.

[10] Nickel JC, True LD, Krieger JN, et al. Consensus development of a histopathological classification system for chronic prostatic inflammation. BJU Int 2001; 87:797-805.

[11] Doble A, Walker MM, Harris JR, et al. Intraprostatic antibody deposition in chronic abacterial prostatitis. Br J Urol. 1990; 65:598605.

[12] Yi FX, Wei Q, Li H, et al. Risk factors for prostatic inflammation extent and infection in benign prostatic hyperplasia. Asian J Androl 2006; 8: 621-627

[13] Klos A, Tenner AJ, Johswich KO, et al. The role of the anaphylatoxins in health and disease. MolImmunol 2009; 46:2753-2766.
[14] Robert G, Descazeaud A, Allory Y, et al. Should we investigate prostatic inflammation for the management of benign prostatic hyperplasia? EurUrolSuppl 2009; 8:879-886.

[15] Minnery CH, Getzenberg RH. Benign prostatic hyperplasia cell line viability and modulation of jm-27 by doxazosin and Ibuprofen. J Urol 2005; 174:375-379.

[16] Di Silverio F, Bosman C, Salvatori M, et al. Combination therapy with rofecoxib and finasteride in the treatment of men with lower urinary tract symptoms (LUTS) and benign prostatic hyperplasia (BPH). EurUrol 2005; 47:72-78.

[17] Falahatkar S, Mokhtari G, Pourreza F, et al. Celecoxib for treatment of nocturia caused by benign prostatic hyperplasia: a prospective, randomized, double-blind, placebo-controlled study. Urology 2008; 72:813-816.

[18] Addla SK, Adeyoju AB, Neilson D, et al. Diclofenac for treatment of nocturia caused by nocturnal polyuria: a prospective, randomised, double-blind, placebo-controlled crossover study. EurUrol 2006; 49:720-725.

[19] Buck AC. Is there a scientific basis for the therapeutic effects of Serenoarepens in benign prostatic hyperplasia? Mechanisms of action. J Urol 2004; 172:1792-1799.

[20] Merendino RA, Salvo F, Saija A, et al. Malondialdehyde in benign prostate hypertrophy: a useful marker? Mediators Inflamm 2003; 12:127-128.

[21] Rohrmann S, De Marzo AM, Smit E, et al. Serum C-reactive protein concentration and lower urinary tract symptoms in older men in the Third National Health and Nutrition Examination Survey (NHANES III). Prostate 2005; 62:27-33.

[22] Liu L, Li Q, Han P, et al. Evaluation of interleukin-8 in expressed prostatic secretion as a reliable biomarker of inflammation in benign prostatic hyperplasia. Urology 2009; 74:340344.

[23] Zhou W, Patel H, Li K, et al. Macrophages from C3-deficient mice have impaired potency to stimulate alloreactive T cells. Blood 2006; 107:2461-2469.

[24] Briganti A, Capitanio U, Suardi N, et al. Benign prostatic hyperplasia and its aetiologies. EurUrolSuppl 2009; 8:865-871. 